\title{
LA SUMMA DIVISIO IURIS PÚBLICO/PRIVADO DE LAS DISCIPLINAS JURÍDICAS
}

\section{Alejandro Vergara BlanCo*}

RESUMEN: En este artículo se analiza la vieja dicotomía Derecho público/Derecho privado, summa divisio de la disciplina jurídica e implica referirse a la epistemología de las disciplinas jurídicas, de donde surge esa otra dicotomía que está en la apertura y en el cierre del fenómeno jurídico: lex y ius. De este clasificatorio fundamental surge no solo una bipolaridad útil para clasificar las especialidades a que se dedican los juristas, sino bases y principios jurídicos que se transmiten a las normas respectivas; y estas bases y principios pueden ser alterados mediante la técnica de la supletoriedad integrando normas entre distintos sectores del Derecho.

PALABRAS CLAVE: Ciencia Jurídica - Derecho público - Derecho privado - lex - ius.

\section{THE PUBLIC/PRIVATE SUMMA DIVISIO IURIS OF THE JURIDICAL DISCIPLINES}

\begin{abstract}
This article analyzes the old dichotomy between public and private Law, summa divisio of the juridical discipline and involves referring to the epistemology of the juridical disciplines, where we find the other dichotomy that deals with the opening and closing of the juridical phenomena: lex and ius. Not only does a useful bipolarity arise from this fundamental classification of subject matters that jurists are dedicated to, but also the basis and juridical principles transmitted to the corresponding norms; and these basis and principles can be amended by means of the supplementary technique integrating norms among different areas of the Law.
\end{abstract}

\footnotetext{
Profesor Titular de Derecho Administrativo, Facultad de Derecho Pontificia Universidad Católica de Chile. Correo electrónico: alvergar@uc.cl

Fecha de recepción: 28 de marzo de 2010.

Fecha de aprobación: 15 de julio de 2010.
} 
KEY WORDS: Juridical Science - Public Law - Private Law - Lex - Ius.

\section{INTRODUCCIÓN}

La bipolaridad público/privado es una summa divisio de las disciplinas jurídicas, que marca un clasificatorio fundamental de todas ellas.

Es tarea de la Teoría del Derecho comprender y analizar el fenómeno del Derecho; en especial, las conexiones entre las normas (lex) y la ciencia del Derecho (ius), y es tarea de la epistemología de las disciplinas jurídicas analizar la manera en que se construyen las ramas y su división bipartita.

En este apartado, analizo la vieja dicotomía Derecho público/Derecho privado, summa divisio disciplinaria jurídica que pareciera siempre estar en medio de todo; e implica referirse a la epistemología de las disciplinas jurídicas, de donde surge esa otra dicotomía que está en la apertura y en el cierre del fenómeno jurídico: lex y ius ${ }^{1}$.

De ahí que cabe una revisión de epistemología de las disciplinas jurídicas: de la summa divisio iuris, que, de una manera heurística, clasifica en público y privado todo el Derecho. De este clasificatorio fundamental surge no solo una bipolaridad útil para clasificar las especialidades a que se dedican los juristas, sino bases y principios jurídicos que se transmiten a las normas respectivas; y estas bases y principios, como veremos, pueden ser alterados mediante la técnica de la supletoriedad ${ }^{2}$, integrando normas entre distintos sectores del Derecho.

\section{1) HisTORIA Y AMPLIA ACEPTACIÓN ACTUAL DE LA BIPOLARIDAD PÚ- BLICO/PRIVADO}

\section{1) LA BIPOLARIDAD EN LA HISTORIA JURÍDICA}

Esta rancia clasificación, que podemos calificar como summa divisio iuris, ha tenido un tránsito singular en la historia del pensamiento jurídi-

1 Ideas y desarrollo ya expuesto, en otro contexto, en Vergara Blanco, Alejandro (2004). "Público y privado ante la dogmática y la teoría del derecho. En especial, las disciplinas de bienes públicos, minas y aguas (a propósito de un reciente libro sobre la "constitucionalización del derecho")", en: Revista de Derecho Público, N 66 (Santiago, Universidad de Chile) pp. 495-523.

2 En especial cabe considerar este trabajo como complementario de Vergara Blanco, Alejandro (2009). "Derecho administrativo y supuesta supletoriedad general del Código Civil", Revista de Derecho Administrativo, No 3, pp. 45-68, en que se analiza la supletoriedad de las normas civiles respecto de casos de derecho administrativo, pues las dificultades de una supletoriedad coherente aumentan en caso de que se aplique desde un sector a otro de la summa divisio del Derecho. 
co: ingresa a la historia en Roma ${ }^{3}$, luego desaparece de escena durante la Edad Media, reapareciendo en la época moderna, sin solución de continuidad hasta hoy.

En efecto, la referida bipolaridad surge a la historia de las fuentes en un fragmento de carácter doctrinario, de las Instituciones de Ulpiano, recogido en las Institutas y en el Digesto ${ }^{4}$. Dicho texto señala que los aspectos del estudio (positiones studii) del Derecho son dos: publicum et privatum:

i) "es derecho público, el que se refiere al estado de la cosa romana" [publicum ius est, quod ad statum rei Romanae spectat]; y

ii) "[es derecho] privado, el que [atañe] a la utilidad de cada individuo" [privatum, quod ad singulorum utilitatem].

Desaparece esta distinción durante la Edad Media, época dominada por el concepto de "Derecho común" (ius commune) y en que impera una noción de ordenamiento jurídico basada en la autonomía, respecto de los derechos particulares (iura propia) ${ }^{5}$. Romanistas y canonistas rehusaron durante muchos años mutilar la unidad del panorama ${ }^{6}$.

Reaparece el díptico Derecho privado/Derecho público a mediados del siglo XVII en la literatura jurídica ${ }^{7}$, en que se compusieron diferentes obras que se titulaban, precisamente, y con total naturalidad, ya de Derecho privado, ya de Derecho público ${ }^{8}$. A partir de esa época, y hasta hoy, la terminología la adoptan con total generalidad los autores de obras jurídicas, para distinguir, parafraseando a Ulpiano, positiones studii iuris, los campos científicos.

Es evidente que con esa misma naturalidad la retoman filósofos ${ }^{9}$, y los estudios de Derecho se clasifican en las escuelas de enseñanza de ese

3 Sobre el origen e historia en Roma, véase: Nocera, Guglielmo (1992). Il binomio pubblicoprivato nella storia del diritto, Napoles: Edizioni Scientifiche Italiana, 208 pp, pp. 7 y ss.

$4 \quad$ Recopilado tanto en Institutas, I, 1, 4, y en Digesto, I, I, 1, 2.

5 Grossi, Paolo (1995). L'ordine giuridico medievale, Roma-Bari: Laterza, p. 226. Existe una traducción al espańol: Grossi, Paolo (1996). El orden jurídico medieval, Madrid: Marcial Pons, 256 pp, p. 224). Lo constata igualmente: Mestre, Jean-Louis (1985). Introduction historique au droit administratif français, París: Presses Universitaires de France, 294 pp., pp. 131, 170-180.

6 Así: Chevrier, G. (1952). "Remarques sur l'introduction et les vicissitudes de la distinction du "jus privatum" et du "jus publicum" dans les œuvres des anciens juristes français", Archives de philosophie du droit, pp. 5-77, p. 7.

7 Chevrier (1952) p. 7.

8 Es el caso del Tratado de Derecho Público de Domat, Jean (1697). Le droit public, suite des lois civiles dans leur ordre naturel, en Euvres de Jean Domat, Paris: Libraire de Jurisprudence, 1829, reed.: Caen, Université de Caen, 1989

9 Véase la Metaphysik der Sitten (1797), de Kant, cuya primera parte está dedicada a los "principios metafísicos de la doctrina [teoría] del derecho", en que ofrece una división suprema del Derecho, que denomina, precisamente: Derecho público y Derecho privado. 
mismo modo; así suele dividirse la enseñanza y los diferentes departamentos o secciones que agrupan a los diferentes profesores de Derecho. Aún más, los especialistas en las distintas ramas del Derecho se suelen autocalificar o de "publicistas" o de "privatistas", si su dedicación es relativa a alguna disciplina de uno de los campos del díptico ${ }^{10}$.

\section{2) LO PÚBLICO Y PRIVADO ANTE EL DERECHO ACTUAL}

La bipolaridad Derecho público/Derecho privado hoy forma parte indesmentible de la cultura jurídica y es una distinción que desde hace mucho tiempo estructura el discurso de los juristas de nuestra tradición "continental"11. Así, el tema es objeto de una intensa discusión, desde los que niegan la distinción ${ }^{12}$ hasta los que la relativizan ${ }^{13}$, pero todo jurista en sus obras pareciera que se siente obligado a señalar algo sobre la distinción, así sea vagamente o con mayor precisión ${ }^{14}$.

Sin perjuicio de ello, lo público/privado hoy no solo es materia de análisis jurídico, sino que es objeto de estudio de otras perspectivas, ya filosóficas, sociológicas o históricas; de ahí que aún muchos piensen que es meramente ideológica y no jurídica. Lo público y privado surge de la

10 Sobre el fenómeno chileno de asignar el papel de un “derecho común” al Código Civil, véase Vergara Blanco, Alejandro (2010). "Un mito jurídico: el Código Civil como supuesto centro del sistema normativo", Revista de Derecho Público, Santiago, Universidad de Chile. (en prensa).

11 Troper, Michel (1994). Pour une théorie juridique de l'État, Paris: Presses Universitaires de France, 358 pp., p. 184. La distinción pareciera no tener relevancia en el Common Law, en el Derecho anglosajón. Véase Oliver, Dawn (2001): “Pourquoi n’y a-t-il pas vraiment de distinction entre droit public et droit prive en Angleterre?", Revue Internationale de Droit Comparé, $\mathrm{N}^{\circ} 2$, pp. 327-338, quien señala que "no existe más que el derecho y la justicia”, y Auby, Jean-Bernard / Freedland, Mark (2004). La distinction du droit public et du droit privé: regards français et britanniques. The Public Law/Private Law Divide: une entente assez cordiale?, Paris: Editions Pánthéon-Assas, 250 pp., que contiene las actas de un encuentro franco-británico de profesores de distintas especialidades, dirigido a analizar la dicotomía en los dos sistemas.

12 Desde una posición normativista que niega la distinción; es el caso de Kelsen.

13 Discusión que no podemos abordar aquí; un panorama y amplia bibliografía en PUGLIATTI, Salvatore (1958). "Diritto pubblico e diritto privatto", voz en: Enciclopedia del diritto, XII, Milán: Giuffrè editore, pp. 606-746.

14 Es posible reenviar a cada manual introductorio de las disciplinas del Derecho, o de filosofía o teoría del Derecho. La bibliografía podría ser amplísima. Para nuestros efectos actuales, podemos constatarlo en: Radbruch, Gustav (1999). Filosofía del Derecho, Granada: Editorial Comares, pp. 159-164; Bоввіо, Norberto (2004). "La gran dicotomía: público/ privado", en él mismo: Estado, Gobierno y Sociedad. Por una teoría general de la política, México: Fondo de Cultura Económica, pp. 11-38; Desmons, Éric (2003). "Droit privé, droit public", voz en: Dictionnaire de la culture juridique, Paris: Quadrige/Lamy-PUF, pp. 520-525. Para Chile, el señero intento de Céspedes Proto, Rodrigo (2004). "La clasificación del derecho en público y privado en el ordenamiento jurídico chileno", Revista Actualidad Jurídica $\mathrm{N}^{\circ}$ 9, pp. 273-300. 
realidad humana, y de la vida en sociedad ${ }^{15}$, y tales miradas son útiles al Derecho, pero no siempre coincidentes. No abordamos esas perspectivas.

Es, entonces, esta bipolaridad una creación de la literatura jurídica y de la enseñanza del Derecho. Es, notoriamente, la incorporación de un lenguaje doctrinario de los autores y profesores, conectado con la realidad, y que poco a poco fue impregnando el diálogo con el resto de la literatura y en especial con el legislador.

En efecto, hoy se torna necesario su análisis ya no solo como un diálogo teórico en medio de la epistemología de las disciplinas del Derecho, sino que se requiere un análisis dogmático-jurídico, esto es, de interpretación de normas vigentes, pues el legislador ha retomado esta clasificación. Es el caso de los dos siguientes ejemplos:

i) el artículo 21 del Decreto con Fuerza de Ley $N^{\circ} 164$, de 1991 [cuyo texto fue fijado por el DS $\mathrm{N}^{\circ}$ 900, de 1996], Ley de Concesiones de Obras Públicas, se refiere a "normas de derecho público" y a "normas de derecho privado;

ii) el artículo $1^{\circ}$ de la Ley No 19.886 , de 2003, de bases sobre contratos administrativos de suministro y prestación de servicios, se refiere a "normas de derecho público" y a "normas de derecho privado"16.

$\mathrm{Al}$ respecto, cabe, entonces, ofrecer las dos siguientes respuestas:

$1^{\circ}$ lo que sea el Derecho público y el Derecho privado; y, enseguida,

$2^{\circ}$ lo que sea una norma de uno u otro sector.

\section{3) LAS FUNCIONES DE LA BIPOLARIDAD}

La claridad sobre la bipolaridad ya no solo es necesaria a efectos de la ciencia del Derecho, para buscar coherencia o similitudes en cada sector del Derecho, para los efectos de su enseñanza, sino también para estos dos efectos prácticos:

15 Véase perspectivas filosóficas sobre la esfera pública y privada en Arendt, Hannah (1958). The Human Condition, Chicago, Illinois: The University of Chicago Press, cap II, pp. $37-$ 95. Existe traducción: La condición humana, Barcelona: Paidós, 1993, 366 pp., y en HABermas, Jürgen (1962). Strukturwandel der Öffentlichkeit [traducc.: Historia y critica de la opinión pública, Madrid, 1982; traducc. francesa: L'espace public. Archéologie de la publicité comme dimension constitutive de la societé bourgeoise, Paris, Payot, 1978], pp. 94-123. Una visión histórica en Clavero, Bartolomé (1991). Razón de Estado, razón de individuo, razón de historia, Madrid: Centro de Estudios Constitucionales, 239 pp.

16 Señala la Ley: "Los contratos (...) se ajustarán a las normas y principios del presente cuerpo legal $y$ de su reglamentación. Supletoriamente, se les aplicarán las normas de Derecho Público y, en defecto de aquellas, las normas del Derecho Privado". 
$1^{\circ}$ asignar tal o cual condición a las normas; y

$2^{\circ} \quad$ verificar si es posible o coherente el método de integración de normas de ambos sectores; esto es, si es teórica y prácticamente aceptable la intromisión de normas para llenar lagunas de uno hacia otro sector ${ }^{17}$.

El breve desarrollo que ofrecemos en este trabajo sobre esta bipolaridad solo intenta cubrir el segundo objetivo señalado.

\section{2) EL FENÓMENO NORMATIVO Y LO PÚBLICO-PRIVADO}

\section{1) EL ORIGEN ÚNICO DE LAS FUENTES NORMATIVAS}

La tarea de la dogmática jurídica presupone, al menos en un inicio, como punto de partida, la aceptación de un ordenamiento jurídico -leyes- como parte del fenómeno jurídico. En nuestra vigente realidad regulatoria, por un dato primigenio, es el populus, reunido en plebiscito, el que dicta la Constitución Política, y tal cuerpo normativo contiene regulaciones (normas: lex), cuya naturaleza solo podemos calificar una vez construidas las disciplinas jurídicas (ius), las que, a su vez, son clasificadas como públicas o privadas.

El legislador (o el constituyente) regula la vida social de acuerdo a imperativos políticos, sin ánimo clasificador; es inimaginable un legislador con conciencia clasificatoria en relación a una naturaleza pública o privada. El legislador simplemente dicta normas, las cuales solo con posterioridad son clasificadas por los juristas en los referidos ámbitos. Las disciplinas del Derecho son las que, en primer término, pueden ser claramente clasificables en privadas o públicas, conformando los dos antiguos brazos del Derecho, como ciencia, por una asentada convención académica; y la transmisión de esta clasificación a las leyes o normas se produce en la operación posterior que hace el jurista, al sistematizar la normativa, integrando una determinada norma como parte de cada disciplina; $y$, en tal caso, el jurista le asigna a cada ley o norma una determinada naturaleza, según su materia (civil, procesal, constitucional, administrativa, laboral, etc.); materia que, a su vez, será pública o privada, según la ubicación de tal disciplina en tal bipartición del Derecho científico. Así, solo una vez recorrido todo ese proceso, esa naturaleza será transmitida por el jurista a la ley o norma.

Suele ocurrir en la práctica legal que un mismo cuerpo normativo puede llegar a incluir normas de distinta naturaleza: ya públicas, ya privadas. Lo que importa a la ciencia del Derecho, para efectos clasificatorios,

17 Tema al que me refiero en Vergara (2009). 
es la sustancia del contenido; el continente (la norma específica; el Derecho/legal) importa a efectos de validez o jerarquía normativa ${ }^{18}$. El origen del Derecho/legal no es público o privado; y si la naturaleza del Derecho legal puede llegar a ser binaria, esto es por una constatación posterior, obra del jurista, como se ha sostenido previamente.

La clasificación público/privado no dice relación con un supuesto origen binario de las fuentes del Derecho positivo vigente, como si fuesen de autores distinguibles ${ }^{19}$; pues hoy todas ellas son originadas del único modo políticamente aceptado: el populus, en vía plebiscitaria (Constitución Política), o el Estado/legislador (el Congreso: casi siempre: leyes (y un sustituto: el derecho consuetudinario); el Presidente de la República: DFL; la Corte Suprema: auto acordados).

De lo anterior surge que los privus de hoy (particulares) nunca son autores de un Derecho/legal, esto es, de regulaciones. Un contrato o un testamento no son leyes, no constituyen fuentes del Derecho/legal, ni se pueden considerar "regulaciones" en ninguna acepción por laxa que sea, sino que son simplemente actos privados, unilaterales o bilaterales, según la clásica clasificación, fruto del principio de la autonomía de la voluntad. De ahí que un jurista actual no puede tomar en serio el art. 1545 del Código Civil, en cuanto se refiere a que el contrato es una "ley" para los contratantes, la que no puede ser considerada sino como una mera metáfora sobre la intangibilidad unilateral de sus cláusulas.

\section{2) LA NATURALEZA JURÍDICA DE CADA NORMA}

A raíz de lo previamente expuesto, a cada una de las ramas o disciplinas del Derecho, esto es, las ciencias especializadas del Derecho, es posible incluirlas en alguno de estos dos polos o esferas: ya en el Derecho público, ya en el Derecho privado. Ahora, el Derecho/legal, esto es, la legislación o cada regulación sectorizada, o incluso cada norma, en verdad, es clasificada o sistematizada "desde" tales disciplinas, y al quedar situado en cada una de esas ramas, según su materia, asume esa naturaleza. Es el científico del Derecho, observando la normativa y la realidad de la vida social que otorga cada norma en su "hipótesis de hecho", el que diseña, separa, disgrega, disecciona, como quiera llamársele, las concentraciones regulatorias o de normas (de cualquier jerarquía: constitucional o legal), y verifica y asigna la naturaleza de tales normas (lex), considerándolas a partir de tal análisis como "fuente" de la disciplina respectiva. Y esta ta-

18 Véase Vergara Blanco, Alejandro (1995). Prólogo a Alejandro Guzmán, Las cosas incorporales en la doctrina y en el derecho positivo (Santiago, Editorial Jurídica de Chile, 1995), pp. 9-15.

19 Como cree verlo Guzmán, Alejandro (2001). El derecho privado constitucional de Chile, Valparaíso: Ediciones Universitarias de la Universidad Católica de Valparaíso, 302 pp., p. 18. 
rea, propia de la "doctrina" (de los juristas), conforma el llamado sistema externo.

En el análisis normativo (del Derecho/legal: primera fase del fenómeno jurídico) parte el trabajo del jurista, creando y delimitando las disciplinas jurídicas y formulando teorías y principios jurídicos; y al conectar una norma con una disciplina, le asigna, a la luz, una naturaleza jurídica. Es lo que explicamos en seguida.

\section{3) PÚBLICO Y PRIVADO DE DISCIPLINAS JURÍDICAS Y LEYES}

El binomio "público-privado" es hoy, antes que nada, una summa divisio disciplinaria; es un gran clasificatorio disciplinario que divide en dos partes toda la constelación de disciplinas autónomas que conforman el universo jurídico científico ${ }^{20}$.

Este binomio no explica el origen de las leyes que componen el orden interno. Es más bien una (en verdad: "la") gran clasificación del fenómeno jurídico dogmático: toda disciplina jurídica debiera ser susceptible de ser sindicada como pública o privada (o, lo que es lo mismo, de Derecho público o de Derecho privado); de otro modo, dejaría de cumplir su rol clasificatorio.

Cabe revisar, entonces, el binomio público/privado tanto en la lex como en el ius. En efecto, la clasificación público/privado es relativa:

i) al contenido de las disciplinas jurídicas (ius); y

ii) desde ahí, en seguida, de manera indirecta, a la naturaleza de las normas (lex).

Analizaremos ambos lugares en que se usa y aplica este binomio público/privado en materia jurídica.

\section{1) BIPARTICIÓN DE TODAS LAS DISCIPLINAS JURÍDICAS}

El binomio se aplica, en primer lugar, al "sistema” jurídico "abstracto-conceptual", elaborado por los juristas.

El objeto clasificado por la bipolaridad público/privado, en este caso, son las disciplinas jurídicas, las que consisten en la especificación de una materia, de la que se deriva la naturaleza de las normas que son su objeto.

¿Cómo se clasifican las disciplinas jurídicas? Estas son reunidas en dos bloques, distinguiendo las disciplinas que son públicas de las que son privadas. Todas las disciplinas del Derecho son clasificadas e incorporadas al interior de cada una de esas dos partes de la summa divisio, y así, cada

\footnotetext{
20 Véase VErgara (2004).
} 
disciplina jurídica (el Derecho Civil, el Derecho Penal, el Derecho Administrativo, el Derecho Laboral, etc.) resulta ser de uno u otro sector.

Como cada disciplina, a su vez, sistematiza las normas relativas a su materia (civil, penal, administrativa, laboral, etc.), como vemos en seguida, esta clasificación público/privado se transmite, indirectamente, a las normas.

Así vemos que la primera aplicación del binomio está dirigida a las disciplinas científicas; al llamado "sistema externo", no legislativo, sino abstracto conceptual; a cada una de esas ciencias especiales, o especialidades a que se dedican los juristas. Es, entonces, en primer término, una clasificación dirigida a los sectores doctrinarios.

\section{2) ASIGNACIÓN DE LA "NATURALEZA JURÍDICA" DE CADA LEY}

El binomio se aplica, también, pero de manera más bien indirecta, al "sistema" jurídico "legal", elaborado por el legislador.

El objeto clasificado por la bipolaridad público/privado, en este caso, son las normas jurídicas.

Recordemos que las normas de una materia dada, en su conjunto, son el objeto de una disciplina determinada que las sistematiza: el Derecho Civil, el Derecho Penal, el Derecho Administrativo, el Derecho Laboral, etc.

¿De qué manera una lex cualquiera se considera de tal o cual disciplina y se incorpora a su núcleo y es estudiada como tal? Se llega a decir que tal o cual ley o norma es civil, penal, administrativa, laboral, etc., a partir del análisis de la realidad jurídica o materia regulada, de lo que surge su "naturaleza jurídica": civil, penal, administrativa, laboral, etc. Entonces, como cada norma tiene una "naturaleza jurídica" asignada, pueden a su vez ser calificadas de normas públicas o privadas, según lo sea la disciplina jurídica cuyo núcleo dogmático esa norma integra.

Estos son los dos enfoques, desde el punto de vista jurídico, para referirse a lo público o privado.

\section{4) LO PÚBLICO O PRIVADO DE LA CIENCIA JURÍDICA}

En suma, el fenómeno de lo privado/público en el Derecho, antes que nada, cabe reducirlo a la clasificación que realizan los científicos del Derecho en dos grandes agrupaciones de disciplinas jurídicas, según su contenido.

$Y$ es respecto de las normas que componen dichas disciplinas, que se realiza el trabajo de disección del jurista: fijando su naturaleza jurídica o formulando principios, instituciones y teorías jurídicas, a través de todo el aparataje técnico propio de la Ciencia del Derecho. Además, como cada disciplina jurídica tiene su propio núcleo, sus bases, principios y 
características esenciales, a partir de ello surge su autonomía disciplinaria; así, existe el Derecho penal (de las penas); laboral (de la relación subordinada); de aguas (del aprovechamiento de las aguas), etc. Estas materias y subsecuentes disciplinas, según sus parentescos forman así dos grandes familias, dando origen a la gran clasificación en público/privado.

\section{1) LAS DISCIPLINAS JURÍDICAS Y SU AUTONOMÍA}

Para la conformación de una disciplina jurídica, y por tanto para considerarla autónoma desde la perspectiva jurídica, existen aspectos esenciales que conforman su contenido: y que el jurista debe descubrir observando los datos normativos y la realidad material; son los aspectos que constituyen el "núcleo dogmático" de cada disciplina, pues en tal núcleo debe ser posible descubrir los institutos jurídicos centrales de cada disciplina autónoma. De estos aspectos esenciales, diseccionados de su regulación (lex), surgen las instituciones nucleares de cada disciplina y los principios generales del Derecho (ius), que permiten una respuesta de ciencia jurídica, con todos sus agregados valóricos, y no de mero legalismo, a los problemas sociales.

Las clasificaciones que realiza la Ciencia jurídica en público/privado y, enseguida, en otras ramas especiales: tributario, forestal, financiero, medio ambiente, etc., están dirigidas a construir disciplinas de estudio, configurando principios jurídicos a partir de los cuales ofrecer modelos de solución; para ello la Ciencia del Derecho "sistematiza" leyes vigentes en un momento determinado. A tales leyes la Ciencia del Derecho les otorga una naturaleza jurídica, y sea donde sea que estén recopiladas, o la denominación que el legislador (o el constituyente) les haya dado, el jurista las "sistematiza" en una disciplina determinada. Así, lo que hace a una disciplina pública o privada es la clasificación doctrinaria.

Las normas en sí mismas siempre tienen una naturaleza; pero esta naturaleza no surge de su pertenencia a un cuerpo legal con una denominación más o menos afortunada dada por el legislador; ni es el legislador el que lo enuncia; tal naturaleza surge del contenido esencial de cada disciplina y de la clasificación que realiza el jurista, según sea que forme parte del núcleo de una disciplina determinada.

\section{2) LOS DOS HEMISFERIOS DEL DERECHO}

Como hemos dicho, de la clasificación derivada de esta summa divisio surge lo que se llama, por una parte, el Derecho público, y, por otra, el Derecho privado. Ni uno ni otro de estos dos grandes sectores constituyen por sí mismos una disciplina, sino que son expresiones clasificatorias para separar dos grandes agrupaciones de disciplinas jurídicas: 


\section{(4.2.1) Las disciplinas de Derecho público}

Su caracterización más acusada es la existencia de potestades de órganos estatales (propias de la autoridad) en la relación jurídica respectiva (es el caso del Derecho Administrativo: potestad administrativa; del Derecho Constitucional o Político: potestades legislativas; del Derecho Procesal: potestades jurisdiccionales o del proceso).

\section{(4.2.2) Las disciplinas de Derecho privado}

Su caracterización más acusada es la existencia de una relación entre privados o particulares (es el caso del Derecho Civil: ya patrimonial, de familia, sucesorio, de los actos y contratos, etc.; del Derecho Penal; del Derecho Laboral; del Derecho Comercial).

La necesaria asignación de las disciplinas jurídicas como un todo al Derecho Público o Privado, podría llamar a dudas a quienes estimen que las fronteras entre Derecho Público y Privado no siempre son nítidas, pero lo que ofrezco en esta clasificación es un criterio: la existencia de potestades públicas en las ramas y relaciones jurídicas en que interviene como sujeto uno de los Poderes del Estado; y la inexistencia de estas potestades en la relaciones de derecho privado, en que los sujetos interactúan en un pie de igualdad. Es un criterio de división de estos dos hemisferios.

\section{(4.2.3) Dos rancios y ambiguos brocárdicos}

Se conecta con lo anterior lo siguiente: suelen considerarse como sendas características de tal clasificación público/privado los dos siguientes brocárdicos o refranes jurídicos, según los cuales:

i) "en Derecho público solo es posible hacer aquello expresamente permitido"; y

ii) "en Derecho privado es posible hacer todo salvo lo expresamente prohibido".

Tales axiomas en realidad no es posible encontrarlos formulados en ninguna fuente, en esos términos; en verdad, tales arcaísmos son unas fórmulas doctrinarias a través de las cuales debemos entender que se intentó tradicionalmente, con un lenguaje que produce confusiones, describir lo que hoy describimos, con mayor precisión, a través de los dos siguientes fenómenos/principios jurídicos, respectivamente:

10 para el Derecho público: de la juridicidad (o legalidad); en verdad, de la necesaria tipicidad de las potestades de actuación de las autoridades; y 
2o para el Derecho privado: de la autonomía ["privada”] de actuación y creación de derechos de los particulares.

Pero en ambos casos, o en ambas esferas, pública y privada, existen regulaciones, ya sea "prohibiendo", ya sea "permitiendo", respectivamente.

Las disciplinas que integran cada uno de los dos grandes sectores en que se divide el Derecho (Derecho público/Derecho privado) comparten entre sí bases esenciales y principios jurídicos; y, viceversa, las disciplinas de diversos sectores no los comparten. De ahí la dificultad de la utilización de técnicas de integración normativa, como es el caso de la supletoriedad, entre disciplinas de los sectores contrapuestos del Derecho ${ }^{21}$.

\subsection{4) Corolario}

10) La bipolaridad Derecho público/Derecho privado es una summa divisio de las disciplinas jurídicas, que marca un clasificatorio fundamental de todas ellas; origina dos sectores diferenciados del fenómeno jurídico. De ahí que las disciplinas que integran cada uno de estos dos sectores comparten entre sí bases esenciales y principios jurídicos; y, viceversa, las disciplinas de diversos sectores no los comparten. De esta summa divisio, según la materia específica de cada disciplina; el jurista, a posteriori, asigna una naturaleza jurídica concordante a cada norma; y a su vez, dependiendo de la ubicación de la disciplina en uno de los dos polos, tales normas podrán ser sindicadas de naturaleza pública o privada.

$2^{\circ}$ ) De ahí la dificultad de la utilización de técnicas de integración normativa, como es el caso de la supletoriedad, entre disciplinas de los sectores contrapuestos del Derecho. Entonces, no parece adecuada, ante lagunas normativas, la utilización de técnicas de integración normativa, como la supletoriedad, entre disciplinas de los sectores contrapuestos del Derecho; y es problemática, pues las normas así integradas lo hacen $\sin$ los ajustes que incorpora el jurista a través del delicado proceso de construcción de principios jurídicos desde y hacia sectores bipolares/contrapuestos, que no comparten bases esenciales ni principios jurídicos entre sí.

\section{BIBLIOGRAFÍA}

- Arendt, Hannah (1958). The Human Condition (Chicago, Illinois, The University of Chicago Press) [traducc.: La condición bumana (Barcelona, Paidós, 1993) 366 pp].

21 Como lo critico en Vergara (2009), trabajo al que me remito. 
- Auby, Jean-Bernard y Freedland, Mark (2004). La distinction du droit public et du droit privé: regards français et britanniques. The Public Law/Private Law Divide: une entente assez cordiale? Paris: Editions Pánthéon-Assas, 250 pp.

- Bоввіо, Norberto (2004). "La gran dicotomía: público/privado", en: Estado, Gobierno y Sociedad. Por una teoría general de la política, México: Fondo de Cultura Económica, pp. 11-38.

- Céspedes Proto, Rodrigo (2004). "La clasificación del derecho en público y privado en el ordenamiento jurídico chileno", Revista Actualidad Juridica $\mathrm{N}^{\circ}$ 9, pp. 273-300.

- Chevrier, G. (1952). "Remarques sur l'introduction et les vicissitudes de la distinction du "jus privatum" et du "jus publicum" dans les œuvres des anciens juristes français", Archives de philosophie du droit, pp. 5-77.

- Clavero, Bartolomé (1991). Razón de Estado, razón de individuo, razón de historia, Madrid: Centro de estudios constitucionales, 239 pp.

- Desmons, Éric (2003). "Droit privé, droit public”, voz en: Dictionnaire de la culture juridique, Paris: Quadrige/Lamy-PUF, pp. 520-525.

- Domat, Jean (1697): Le droit public, suite des lois civiles dans leur ordre naturel, en: Euvres de J. Domat, Paris: Libraire de Jurisprudence, 1829. [reed.: Caen, Université de Caen, 1989].

- Grossi, Paolo (1995). L'ordine giuridico medievale, Roma-Bari: Laterza) [traducc.: El orden juridico medieval, Madrid: Marcial Pons, 1996, 256 pp.].

- Guzmán Brito, Alejandro (1976). "Notas sobre el derecho público”, Revista de Derecho Público, No 19-20, 1976, pp. 385-393 = "Discurso del Decano...", en: Actas de las VII Jornadas de Derecho público, Valparaíso, 1976, pp. 15-24.

- (2001). El derecho privado constitucional de Chile (Valparaíso: Ediciones Universitarias de Valparaíso [de la Universidad Católica de Valparaíso], 302 pp.

- Habermas, Jürgen (1962). Strukturwandel der Öffentlichkeit [traducc.: Historia y crítica de la opinión pública, Madrid, 1982; traducc. francesa: L'espace public. Archéologie de la publicité comme dimension constitutive de la societé bourgeoise, Paris: Payot, 1978].

- Mestre, Jean-Louis (1985). Introduction historique au droit administratif français, París: Presses Universitaires de France, 294 pp.

- Nocera, Guglielmo (1992). Il binomio pubblico-privato nella storia del diritto, Napoles: Edizioni Scientifiche Italiana, 208 pp.

- Oliver, Dawn (2001). "Pourquoi n'y a-t-il pas vraiment de distinction entre droit public et droit prive en Angleterre?", Revue internationale de droit comparé, $\mathrm{N}^{\circ} 2$, pp. 327-338.

- Pugliatti, Salvatore (1958). "Diritto pubblico e diritto privatto", voz en: Enciclopedia del diritto, XII (Milán, Giuffrè editore) pp. 606-746. 
- Radbruch, Gustav (1999). Filosofía del derecho, Granada: Editorial Comares.

- Troper, Michel (1994). Pour une théorie juridique de l'État, Paris: Presses Universitaires de France, 358 pp. [traducc.: Por una teoría jurídica del Estado, Madrid: Dykinson, 2001, 340 pp.

- Vergara Blanco, Alejandro (1995). Prólogo a Alejandro Guzmán, Las cosas incorporales en la doctrina y en el derecho positivo, Santiago: Editorial Jurídica de Chile, pp. 9-15.

- _ (2001). "La summa divisio de los bienes y recursos naturales ante la Constitución de 1980", en VV.AA 20 años de la Constitución chilena 1981-2001, Santiago: Ediar-ConoSur, pp. 369-390; también en: Ius Publicum No 12 (2004) pp.105-126.

(2002). "Las aguas como bien público (no estatal) y lo privado en el derecho chileno: evolución legislativa y su proyecto de reforma", en: Embid Irujo, Antonio (dir.), El derecho de aguas en Iberoamérica y España: Cambio y modernización en el inicio del Tercer Milenio, Madrid: Civitas, tomo II, pp. 179-213; también en: Revista de Derecho Administrativo Económico, vol. IV No 1, pp. 63-79.

- (2003): recensión al libro de D’Ors, Álvaro, La posesión del espacio, en: Revista Chilena de Derecho, No 2, pp. 415-418.

- (2004). "Público y privado ante la dogmática y la teoría del derecho. En especial, las disciplinas de bienes públicos, minas y aguas (a propósito de un reciente libro sobre la "constitucionalización del derecho"), Revista de Derecho Público, N66, pp. 495-523.

- (2009). "Derecho administrativo y supuesta supletoriedad general del Código Civil”, Revista de Derecho Administrativo No 3 pp. 45-68; también en: La primacia de la persona. Estudios en homenaje al profesor Eduardo Soto Kloss (coordinadores: Jaime Arancibia M. y José Ignacio Martínez E., Santiago: LegalPublishingAbeledo Perrot, en coedición con la Universidad de los Andes, pp. 259-280.

- (2010): "Un mito jurídico: el Código Civil como supuesto centro del sistema normativo", en prensas, Revista de Derecho Público. 